\title{
Modulation of intestinal environment by prebiotic germinated barley foodstuff prevents chemo-induced colonic carcinogenesis in rats
}

\author{
OSAMU KANAUCHI ${ }^{1}$, KEIICHI MITSUYAMA ${ }^{2}$, AKIRA ANDOH $^{3}$ and TOSHIHIKO IWANAGA ${ }^{4}$ \\ ${ }^{1}$ Central Laboratories for Frontier Technology, Kirin Holdings Co. Ltd., 1-13-5 Fukuura, Kanazawa-ku, \\ Yokohama 236-0004; ${ }^{2}$ Kurume University School of Medicine, 67 Asahi-machi, Kurume, Fukuoka 830-0011; \\ ${ }^{3}$ Shiga University of Medical Science, Tsukinowa, Seta, Otsu, Shiga 520-2192; ${ }^{4}$ Hokkaido University Graduate \\ School of Medicine, Laboratory of Histology and Cytology, Kita 15, Nishi 7, Sapporo 060-8638, Japan
}

Received April 3, 2008; Accepted May 5, 2008

DOI: 10.3892/or_00000076

\begin{abstract}
Butyrate was shown to have a preventive effect on colon cancer in vivo. Germinated barley foodstuff (GBF) was in a prebiotic stage and had the potency to attenuate mucosal inflammation and to increase fecal butyrate production in colitis. This study aimed to determine whether the GBF treatment in a colon cancer model had the potency to suppress colon cancer. After a pre-feeding of either a control or a GBF diet for two weeks, male F344 rats received subcutaneous injections of azoxymethane twice, at a dose level of $15 \mathrm{mg} / \mathrm{kg}$ body weight. The injections were administered once a week for 2 weeks ( $n=10 /$ group). Four weeks after that, the number of aberrant crypt foci (ACF) and heat shock protein (HSP) 25-positive cells in colonic mucosa were observed histologically. The mRNA level of slc5a8 was evaluated by in situ hybridization. Colonic mucosal $\beta$-catenin was determined by Western blotting. Cecal short chain fatty acids, B-glucosidase and $B$-glucuronidase were also determined. The results showed that GBF treatment significantly decreased the number of $\mathrm{ACF}$ and $\beta$-catenin formations in the colonic mucosa. GBF significantly increased the production of slc5a8, which is a tumor suppressor gene, as well as the cecal butyrate content and $\beta$-glucosidase activity. $\beta$-glucuronidase activity remained at the same level in GBF and control subjects. The number of HSP25-positive cells in GBF was higher than that in the control group, although it did not reach significant difference. In conclusion, GBF showed anti-tumorigenicity in the AOM rat model. Changes in the colonic environment featured through the increase of butyrate production were found. Although a more detailed study is required, this study
\end{abstract}

Correspondence to: Dr Osamu Kanauchi, Central Laboratories for Frontier Technology, Kirin Holdings Co. Ltd., 1-13-5 Fukuura, Kanazawa-ku, Yokohama 236-0004, Japan

E-mail: kanauchio@kirin.co.jp

Key words: colon cancer, slc5a8, butyrate,, -catenin, microbiota showed the promising anti-neoplastic effects of prebiotic treatment.

\section{Introduction}

Microbiota are shown to have the potency to influence gastrointestinal disease including colorectal cancer and chronic inflammatory disorder (1-5). This means that the food, including probiotics and/or prebiotics can change the colonic microbiota and may prevent diseases such as colorectal cancer or inflammatory bowel disease (IBD) $(2,3)$. The elaborative mechanism by which probiotics and/or prebiotics exert anti-tumorigenic effects remains unknown. However, it is postulated that modifying colonic $\mathrm{pH}$, increases short chain fatty acid production, stimulating immune cells in mucosa (epithelium and lamina propria), and increasing competition for the absorption of nutrients (4).

Recently, modulating the intestinal microbiota has been attempted by the use of prebiotics or probiotics. Germinated barley foodstuff $(\mathrm{GBF})$ is a heterogeneous mixture of insoluble protein and dietary fiber, which is known to function as a prebiotic in the intestine and is reported to be effective in colitis $(6,7)$. These alterations may act beneficially, in part by causing a luminal induction of short chain fatty acids (SCFAs), which are important nutrients for the intestine, and induce an acidic environment (8). In our previous studies, GBF had a therapeutic effect on patients with active ulcerative colitis (UC) and prolonged the remission periods in inactive UC patients $(6,7)$. It is reported that butyrate acts as an antitumor agent by inducing apoptosis in vitro $(9,10)$. Although it is still controversial whether butyrate has a preventive effect on colon cancer in vivo, studies have shown that short chain fatty acids including butyrate have been effective. However, it was also reported that butyrate did not have the potency to produce cancer-preventing effects in vivo $(11,12)$. Since butyrate produced by microbiota is kept in a stable physiological concentration in the entire colon, prebiotic treatment may be a useful medical option for reducing the risk of colon cancer in longstanding-colitis (13).

It was reported that the sodium/glucose co-transporter (slc5a8), a tumor suppressor gene, which codes for mono- 
carboxylates including short chain fatty acids, lactate and pyruvate, was down-regulated in colon cancer $(14,15)$. High levels of slc5a8 methylation were also observed in colon cancer. However, the coexistence of unmethylated slc5a8 in colon cancer cell lines had the potency to manifest an anticolony forming ability (14). Changing the intestinal condition, e.g. short chain fatty acid content, may contribute to the suppression of colon cancer via slc5a8 (16).

In this study, we used the azoxymethane (AOM)-induced colonic carcinogenesis model, which is widely used and features the clinical and pathological characteristics found in human colon cancer, and which induces hyperproliferation in the premalignant phase (17-19). This study aimed to determine whether the GBF treatment in the colon cancer model had the potency to suppress and/or prevent experimental colon cancer as well as act as a modulator in the colonic environment.

\section{Materials and methods}

Composition of GBF and reagents. GBF mainly consists of the aleurone and scutellum fractions of germinated barley obtained by milling and sieving. The dietary fiber fraction of GBF contains primarily low-lignified hemicellulose rich fiber (35\% by weight) and insoluble glutamine-rich protein ( $48 \%$ by weight). During germination, GBF exhibits a conspicuously high capacity for holding water (20).

The protein assay kit was purchased from Pierce (BCA ${ }^{\mathrm{TM}}$ protein assay kit; Rockford IL, USA). All dietary components were purchased from Oriental Yeast Co. Ltd. (Tokyo, Japan). Experimental reagents were purchased from Sigma Aldrich Japan (Tokyo, Japan) and Wako Pure Chemicals (Osaka, Japan).

Animals and diets. Twenty 5-week-old male Fischer 344 rats were purchased from Charles River Japan (Kanagawa, Japan). The rats were housed individually in cages in a room kept at $20-25^{\circ} \mathrm{C}$ and at a level of $40-70 \%$ relative humidity with a $12-\mathrm{h}$ lighting cycle (20). The rats were allowed free access to food and drinking water. The experiment was approved by the Kirin Holdings ethics committee for animal experimentation. The 20 rats were fed laboratory chow for 1 week during their acclimatization period. Then we started the experiments, and the rats were divided into two groups ( $n=10 /$ group). One was a controlled diet group, and the other was the GBF diet group. The total volume of protein and dietary fiber in the two diets was adjusted to $14.6 \%$ protein and $3.0 \%$ dietary fiber, respectively $(20,21)$. The composition of the two diets is shown in Table I.

Experiment 1. After two weeks of pre-feeding using the respective experimental diets, the rats were administered subcutaneous injections of AOM twice, at a dosage level of $15 \mathrm{mg} / \mathrm{kg}$ body weight once a week for two consecutive weeks (17). After the second AOM injection, the rats were given their respective diets for 4 more weeks ad libitum and were sacrificed. Changes in their body weight and food intake are shown in Fig. 1.

ACF analysis. The entire colon was obtained for histological observation including the counting of aberrant crypt foci (ACF). Mucosal manifestations of Slc5a8 were carried out. The rectum at $\sim 3 \mathrm{~cm}$ from the pectinate line was fixed
Table I. Composition of respective diets.

\begin{tabular}{lrr}
\hline & Control & GBF \\
\hline & \multicolumn{2}{c}{$(\mathrm{g} / \mathrm{kg} \mathrm{diet})$} \\
Casein & 100.0 \\
Vitamin mixture $^{\mathrm{a}}$ & 146.0 & 10.0 \\
Mineral mixture $^{\mathrm{a}}$ & 35.0 & 35.0 \\
Choline chloride $_{\text {Cellulose }}$ & 2.0 & 2.0 \\
GBF & 30.0 & \\
Corn oil & & 100.0 \\
Corn starch & 50.0 & 50.0 \\
\hline
\end{tabular}

${ }^{\mathrm{a} A I N}$ 93G formula (20).

with $4 \%$ buffered formalin and stained with $0.2 \%$ methylene blue. According to the methods from the previous report, the ACF were scored under a light microscope (Axioskop40 and AxioVision, Japan) with an area of $2 \mathrm{~cm}^{2}$ of colon being quantified at X100 magnification (17). ACF were distinguished from normal crypts by their increased size, more prominent epithelial cells and their increased pericryptal area.

Organic acid analysis of cecal content. The organic acid content of cecal content in rats fed with the respective diets was determined and modified according to Araki et al (22). Briefly, $0.2 \mathrm{~g}$ of cecal content was weighed, $1.0 \mathrm{ml}$ of Milli-Q water was added and the content was then incubated at $4^{\circ} \mathrm{C}$ for $30 \mathrm{~min}$. After being centrifuged at $12000 \mathrm{rpm}$, at $4^{\circ} \mathrm{C}$, for 10 min, supernatant was obtained, and continuously filtered by using a $0.22 \mu \mathrm{m}$ filter. The organic acids were separated with a Shim-pack SPR-H 250L (Shimadzu Co. Ltd., Kyoto, Japan) The mobile phase was $4 \mathrm{mM}$ of p-Toluene sulfonic acid. The detector was electric conductivity (Shimadzu CDD-6A, Kyoto Japan).

Detection of colonic slc5a8 expression. According to a previous study by Iwanaga et al, in situ hybridization was performed. Briefly, three non-overlapping 45-mer antisense oligonucleotide probes, complementary to the sequences: 358-402, 1491-1535 and 2993-3337 of sodium-dependent monocarboxylate transporter mRNA, were synthesized and labeled with ${ }^{33} \mathrm{P}-\mathrm{dATP}$. Fresh-frozen sections $(10-\mu \mathrm{m})$ were fixed with $4 \%$ paraformaldehyde in a $0.1 \mathrm{M}$ phosphate buffer for $15 \mathrm{~min}$, and acetylated with $0.25 \%$ acetic anhydride in 0.1 $\mathrm{M}$ of triethanolamine- $\mathrm{HCl}(\mathrm{pH} 8.0)$ for $10 \mathrm{~min}$. Hybridization was performed at $42^{\circ} \mathrm{C}$ for $10 \mathrm{~h}$ by adding $10000 \mathrm{cpm} / \mu 1{ }^{33} \mathrm{P}-$ labeled oligonucleotide probes. After being washed, sections were exposed to BioMax MR (Kodak, Rochester, NY) for 10 days $(23,24)$. A detection of intensity was performed by using an Image Gauge (LAS1000, Fujifilm, Tokyo Japan). The intensity of slc5a8 was expressed as the value of the densitometry units.

Experiment 2. As described in Experiment 1, after two weeks of the pre-feeding of the respective diets, the rats received subcutaneous injections of AOM twice, at a dosage level of $15 \mathrm{mg} / \mathrm{kg}$ body weight once a week for two consecutive weeks. After the second AOM injection, the rats were given 


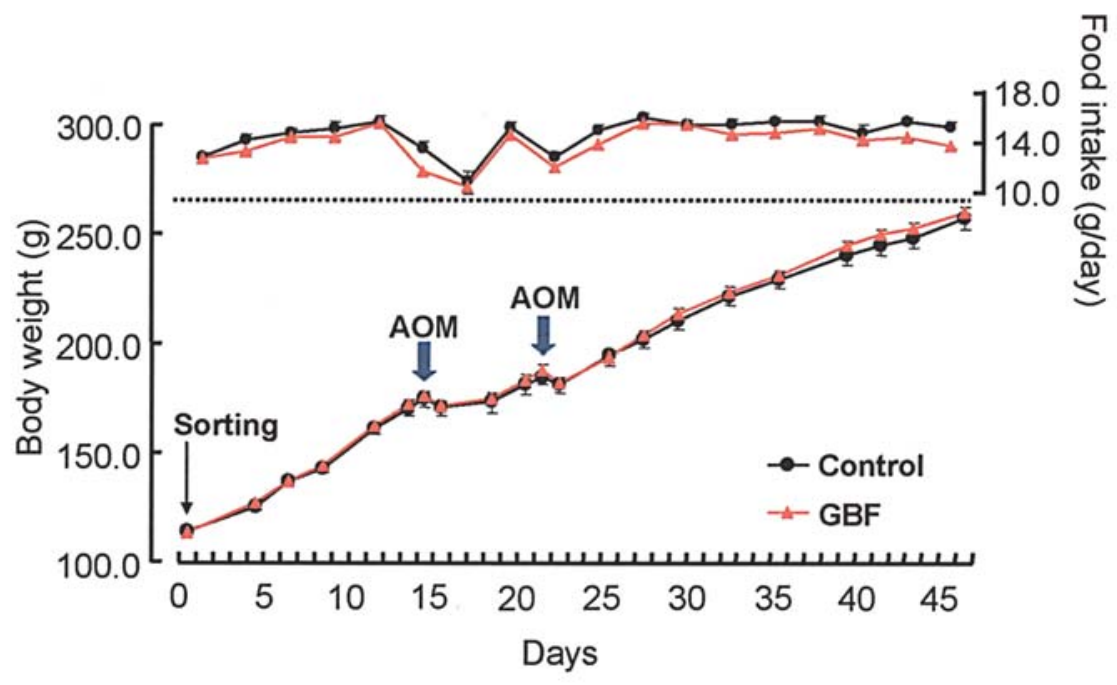

Figure 1. Change in body weight and food intake in rats fed either the control or the GBF diet (Experiment 1). The arrows show the injection of azoxymethane $(\mathrm{AOM})$ s.c. The data are expressed as mean changes \pm SE. No significant differences in body weight and food intake were detected between the two groups during the experimental period.

their respective diets for 4 more weeks ad libitum. Then, 5 rats in each respective group were sacrificed and the remaining rats were euthanized an additional week later.

ACF analysis. Using 5 rats from each of the respective groups, the entire colon was obtained for ACF counting. The colon was fixed with $4 \%$ buffered formalin and stained with $0.2 \%$ methylene blue. As in the previous report, the number of $\mathrm{ACF}$ in the entire colon was counted under a light microscope at x100 magnification. The ACF were distinguished from normal crypts by their increased size, more prominent epithelial cells and their increased pericryptal area. The number of ACF and the number of aberrant crypts (ACs) per rat were quantified (17).

Western blotting of $\beta$-catenin. For the assaying of the Western blotting of $\beta$-catenin of the colonic mucosa, the colon was cut into 2-mm samples and washed with ice-chilled PBS. The cytoplasmic fraction of colonic mucosa was extracted according to the method of Fujise et al (25). After being centrifuged (136000 g x $30 \mathrm{~min}$ ), the supernatant was analyzed through immunoblotting. Equal quantities $(25 \mu \mathrm{g}$ protein $/ \mathrm{ml})$ of protein were electrophoresed in an SDS-PAGE. Thereafter, they were electroblotted onto a polyvinyliden difluoride membrane (Immobilon-P, Millipore, MA, USA). After being blocked with Blockace (Dainippon Sumitomo Pharma, Osaka, Japan), the membrane was incubated with primary antibodies. The monoclonal anti- $\beta$-catenin antibody (610153, BD Biosciences Japan, Tokyo, Japan), the anti-ßactin antibody (A5441, Sigma, MO, USA), and polyclonal goat anti-mouse Ig/HRP (P0447, Dakocytomation, Denmark) were used. The detection kit (ECL Plus Western blotting detection system) was purchased from GE Healthcare (Buckinghamshire, UK). The detection of chemiluminescence was performed by using an Image Gauge (LAS1000, Fujifilm, Tokyo Japan). The intensity of $\beta$-catenin was expressed as the value of the adjusted signal intensity with $\beta$-actin densitometry units. Results are shown as the relative value of the control group. $\beta$-glucuronidase and $\beta$-glucosidase activity of cecal content. Cecal content $(0.3 \mathrm{~g})$ was diluted with $1.0 \mathrm{ml}$ of icechilled PBS (pH 7.4) and shaken (TS-100, Thermal Kagaku Sangyo Co., Tokyo, Japan) for $30 \mathrm{~min}$ at $4^{\circ} \mathrm{C}$. After shaking, it was centrifuged $\left(15000 \mathrm{rpm}, 10 \mathrm{~min}, 4^{\circ} \mathrm{C}\right)$ and the supernatant was immediately filtered by using an Ultra free-MC (at $10000 \mathrm{rpm}, 10 \mathrm{~min}, 4^{\circ} \mathrm{C}$, Millipore, MA, USA). The activity of $\beta$-glucuronidase and $B$-glucosidase was determined by a modified method measuring the release rate of $\mathrm{p}$-nitrophenols from p-nitrophenylglucuronide (Sigma) and p-nitrophenylglucopyranoside (Sigma), respectively (26). The data were measured in the units: $\mu \mathrm{mol} / \mathrm{min} / \mathrm{g}$ cecal content.

Heat shock protein 25 immunostaining assay. The rat and murine forms of HSP27 are slightly smaller than the human form, and for that reason we described their HSP as HSP25 in this study (27). When using the other 5 rats from the respective groups, the entire colon was removed, cut and rinsed with ice-chilled PBS. The distal colon, $8 \mathrm{~cm}$ upward from anus, was then cut off. The colorectum was longitudinally cut into two equal parts. One was mounted on cardboard and fixed in buffered formalin for the HSP25 immunostaining analysis and the other was weighed and immediately used for Western blotting of the $\beta$-catenin in the colonic mucosa. Colon sections were embedded in paraffin and $4 \mu \mathrm{m}$ crosssections were cut. According to the methods described by Ren et al (27), sections were stained for HSP25. The LSAB2 kit (K0609, Dakocytomation) and primary antibody, rabbit polyclonal antibody HSP27, which was purchased from Abcam (ab5579, Abcam Japan, Tokyo, Japan), were used. The number of stained cells per view was counted under a light microscope at $\mathrm{x} 200$ magnification for 6 different areas $(2$ views were made in the distal, middle and proximal part of the colon, respectively), and the results are shown as the mean value per view.

Statistical analysis. Values are presented as the mean \pm SEM. Comparisons of data were made by Student's t-test or the $\chi^{2}$ analysis. Differences were considered significant at $\mathrm{P}<0.05$. 
Table II. The number of aberrant crypt foci in colonic mucosa.

\begin{tabular}{lcccccc}
\hline & Incidence & $1 \mathrm{AC} / \mathrm{ACF}$ & $2 \mathrm{ACs} / \mathrm{ACF}$ & $3 \mathrm{ACs} / \mathrm{ACF}$ & $>4 \mathrm{ACs} / \mathrm{ACF}^{\mathrm{b}}$ & Total ACs/ACF $^{\mathrm{c}}$ \\
\hline Control & $(5 / 5)$ & $57.60 \pm 6.10$ & $64.60 \pm 6.10$ & $26.80 \pm 1.11$ & $17.80 \pm 3.02$ & $2.04 \pm 0.06$ \\
$\mathrm{GBF}$ & $(5 / 5)$ & $57.60 \pm 6.10$ & $64.60 \pm 6.10$ & $26.80 \pm 1.11$ & $17.80 \pm 3.02$ & $2.04 \pm 0.06$ \\
P-value & & 0.037 & 0.006 & 0.089 & 0.013 & 0.119 \\
\hline
\end{tabular}

${ }^{\mathrm{a}}$ Aberrant crypt, ${ }^{\mathrm{b}}$ counted the number of aberrant crypts/ACF as 4 and ${ }^{\mathrm{c}}$ mean number of aberrant crypt/one focus .

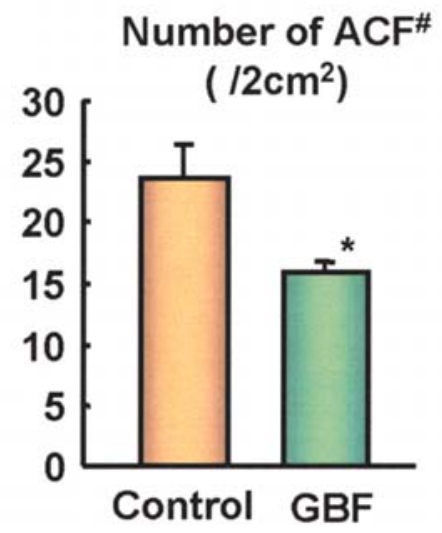

\# Aberrant crypt foci

Figure 2. The number of abberant crypt foci (ACF) were counted under a light microscope, measured per $2 \mathrm{~cm}^{2}$ of colon and were quantified at x100 magnification. The data are expressed as mean changes \pm SE. ACF in the GBF group were significantly lower than that in the control group. ${ }^{*} \mathrm{P}<0.05$ for the difference between the control and GBF.

\section{Results}

Experiment 1. Fig. 1 shows the changes in the body weight, food intake and schedule of AOM administrations. During the experimental period, there were no significant differences in body weight and food intake between the two groups. The number of $\mathrm{ACF}$ in the $2 \mathrm{~cm}^{2}$ parts of the colon is shown in Fig. 2. After the GBF treatment, the number of ACF was

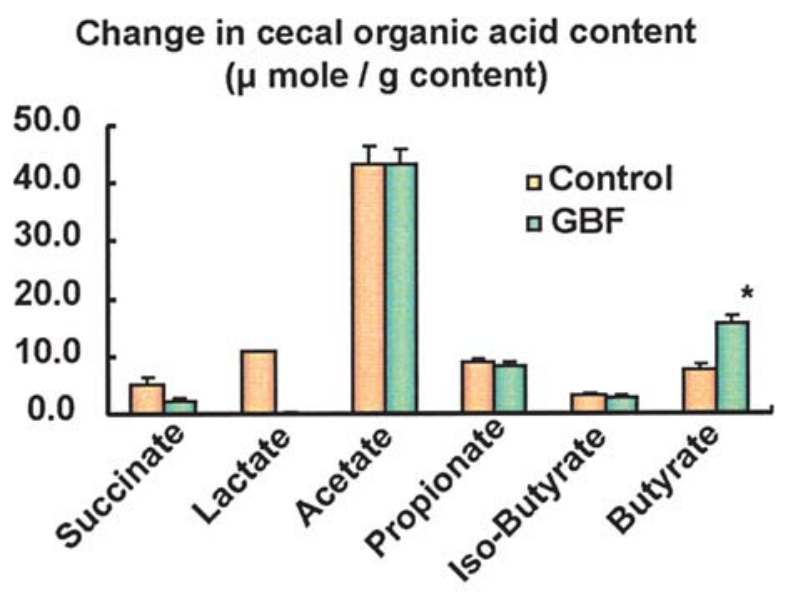

Figure 3. Concentrations of cecal organic acids in rats fed with the control or the GBF diet (Experiment 1). The data are expressed as mean changes $\pm \mathrm{SE}$. Butyrate content was significantly higher in the GBF group than that of the control group, and the succinate content was lower in the GBF group than that of the control group ( $\mathrm{p}=0.057$ ). ${ }^{*} \mathrm{P}<0.05$ for the difference between the control and GBF.

significantly lower than that of the control group. Fig. 3 indicates the change in the cecal organic acid content of the two groups. The GBF treatment showed a significant decrease in the production of succinate and an increase in the amount of butyrate, compared to the control group. Slc5a8 was detected in the distal colon in the two groups. However, the amount of slc5a8 in the GBF group was significantly higher than that of the control group (Fig. 4).

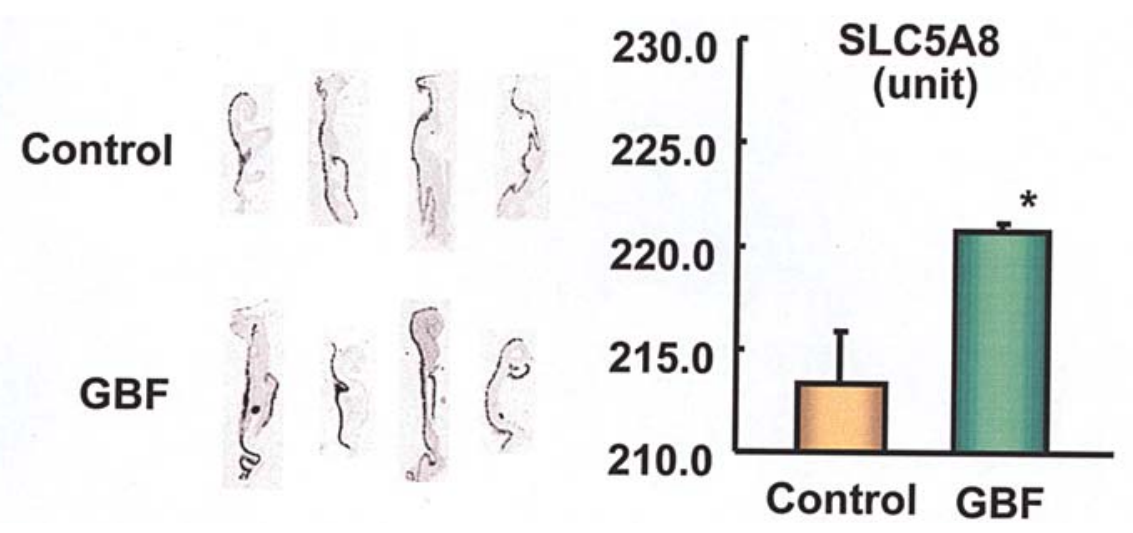

Figure 4. Left, colonic mucosal hybridization of slc5a8 labeled as ${ }^{33} \mathrm{P}-\mathrm{dATP}$. Right, the intensity of slc5a8, and the expression of slc5a8 in the GBF group being significantly higher than that of the control group. The data are expressed as mean changes $\pm \mathrm{SE}$. * $\mathrm{P}<0.05$ for the difference between the control and GBF. 


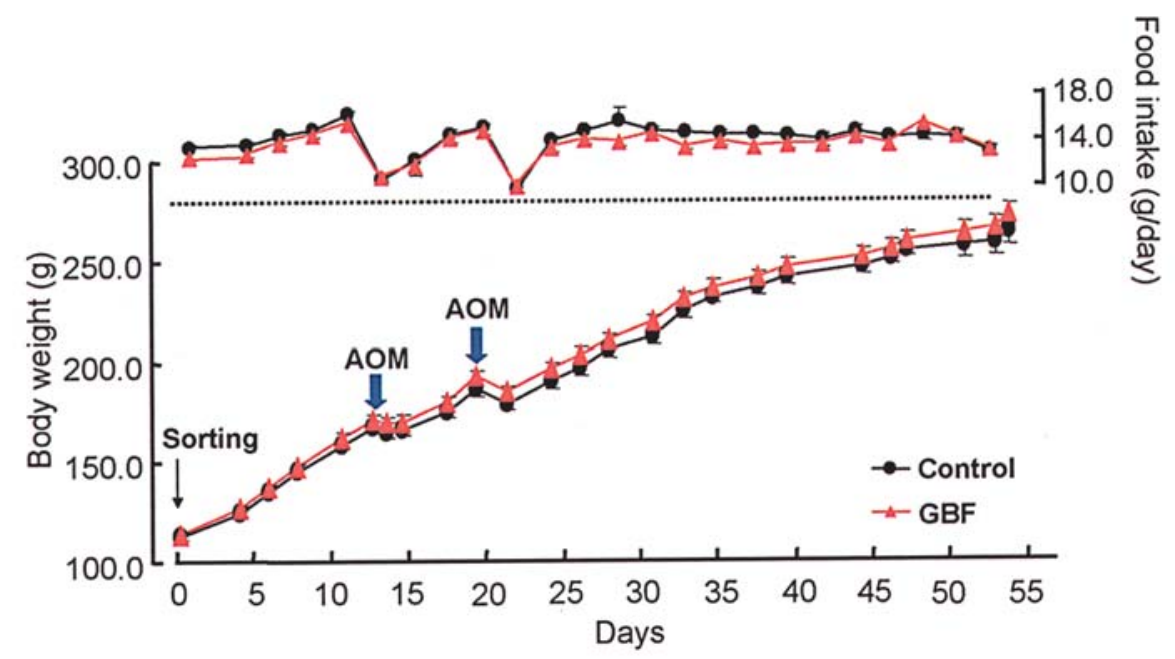

Figure 5. Changes in body weight and food intake in rats fed either the control or the GBF diet (Experiment 2). The arrows show the injection of azoxymethane (AOM) s.c. The data are expressed as mean changes \pm SE. No significant differences in body weight and food intake were detected between the two groups during the experimental period.

\section{Total number of $\mathrm{ACF}$}

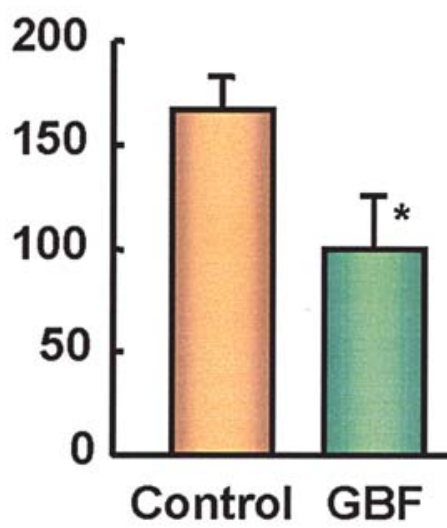

\section{Total number of aberrant crypts/ focus}

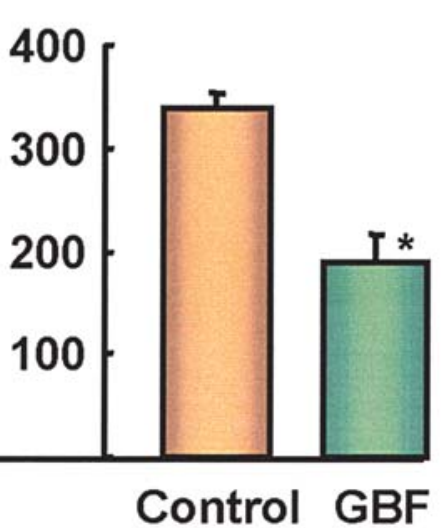

Figure 6. The number of abberant crypt foci (ACF) were counted under a light microscope in the entire colon and the total number of aberrant crypts per focus were quantified at x100 magnification. The data are expressed as mean changes \pm SE. ACF and the total number of aberrant crypts per focus in the GBF group were significantly lower than that of the control group. ${ }^{*} \mathrm{P}<0.05$ for the difference between the control and GBF.

Experiment 2. Fig. 5 shows changes in the body weight, food intake and schedule of AOM administrations. During the experimental period, there were no significant differences in body weight and food intake between the two groups, which was also the case in Experiment 1. The number of ACF in the entire colon ( $n=5 /$ group) is shown in Fig. 6 and Table II. $\mathrm{ACF}$ were caused to form in the rats, and the ACs per ACF were lower in the GBF treatment group than in the control group. Although the number of ACs per ACF was high in the control group, it did not reach a significant difference. The total number of ACF and ACs was significantly lower in the GBF treatment in comparison with the control group levels. Fig. 7 shows the typical appearance of a normal crypt and ACF.

The manifestation of colonic mucosal $B$-catenin is shown in Fig. 8, along with the significant decrease of $\beta$-catenin in the GBF treatment compared with that of the control group.

The activity of $\beta$-glucosidase and $\beta$-glucuronidase in cecal content is shown in Fig. 9. B-glucuronidase activity in the two groups was at the same level, but $\beta$-glucosidase activity in the GBF group was significantly higher than that of the control group. The cecal organic acid content in the two groups is shown in Fig. 10. The GBF treatment significantly increased acetate and butyrate content, in comparison with the control group. Fig. 11 shows a microscopic view of HSP25positive cells in the colonic mucosa $(\mathrm{x} 200)$ and the number thereof. Although the number of HSP25-positive cells per view did not reach a significant difference between the two groups, the GBF treatment showed an increase in the number of cells comparatively.

\section{Discussion}

GBF is a safe prebiotic for UC patients and it may be useful to continue ingestion in order to reduce the risk of colorectal cancer without causing adverse effects produced by anticancer or anti-inflammatory drugs. GBF administration induced 


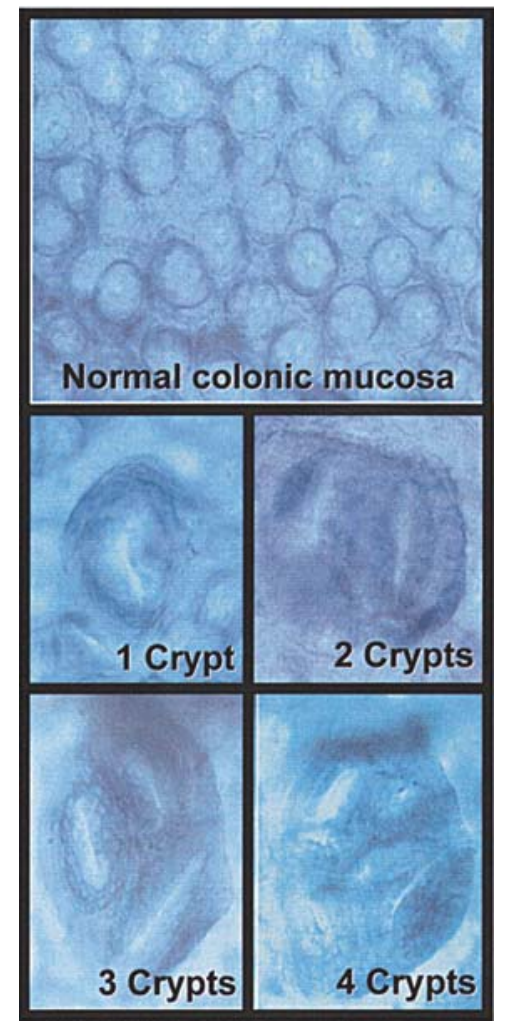

Figure 7. Typical aberrant crypts. ACF were distinguished from normal crypts by their increased size, more prominent epithelial cells and their increased pericryptal area.

an increase in the luminal short chain fatty acid concentration, especially butyrate, which is produced abundantly in the colon by bacterial fermentation of residual food fractions (e.g. dietary fiber) $(28,29)$. One of the promising mechanisms of GBF causing the anti-colitis effect may be due to the increased level of butyrate. The butyrate from GBF significantly suppressed the NFKB activation and phosphorylation of STAT3, which sequentially reduced IL-6 production in the

\section{Expression of $\beta$-catenin}

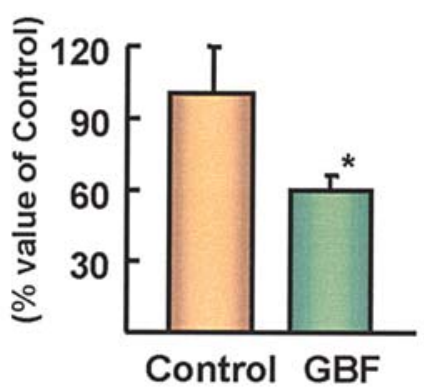

Figure 8. ß-catenin expression in colonic mucosa. The intensity of ß-catenin in the GBF group was significantly lower than that of the control group. The data are expressed as mean changes $\pm \mathrm{SE}$. ${ }^{*} \mathrm{P}<0.05$ for the difference between the control and GBF.

colitis model (30). It was also reported that butyrate possesses the potency to act as an energy source for the epithelium and cause an increase of cell differentiation while suppressing the proliferation of tumor cells through the induction of apoptosis $(31,32)$. Thus, it is considered that the increase of luminal short chain fatty acids may contribute to the reinforcement of epithelial integrity which suppresses mucosal damage.

The incidence of ACF in colonic mucosa in the two groups was exactly the same. However, the total number of $\mathrm{ACF}$ and ACs was significantly lower in the GBF group compared to that of the control group. In general, colon cancer was understood to arise in a multi-step process of histopathological and molecular changes that transform a normal colonic epithelium into invasive colorectal cancer, accompanied by the processes of hyperproliferative epithelium (aberrant crypt foci), small adenoma, large adenoma and colon carcinoma (33). Although more detailed studies are needed, our data suggest that GBF affects the early stages of the pathogenesis of colon cancer, and facilitates the prevention of transforming hyperproliferative epithelia. It was reported

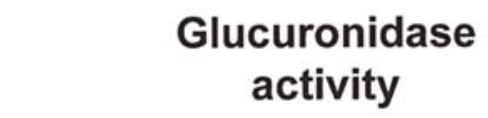

\section{Glucosidase activity}

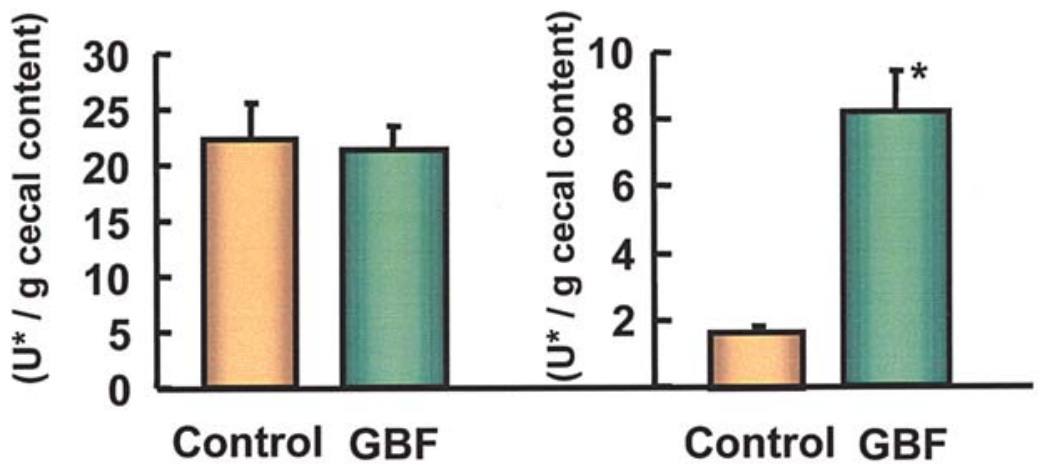

\section{*(U; umol p-nitrophenol released / min)}

Figure 9. Bacterial glycolytic enzyme activities ( $\beta$-D-glucuronidase and $\beta$-D-glucosidase) in cecal content of rats fed the control and the GBF diet. Glucosidase activity in the GBF group was significantly higher than that of the control group, and glucuronidase activity of the respective groups was at the same level. The data are expressed as mean changes \pm SE. ${ }^{*} \mathrm{P}<0.05$ for the difference between the control and GBF. 


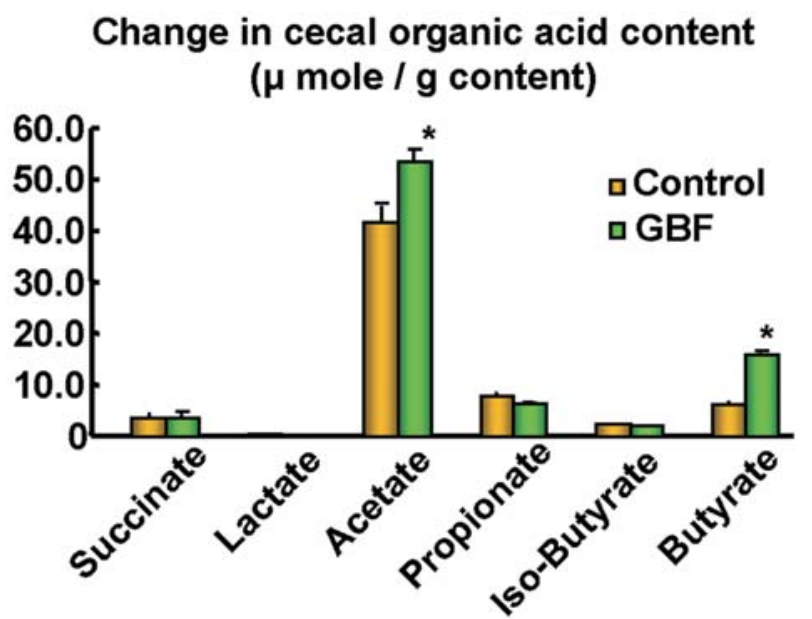

Figure 10. Concentrations of cecal organic acids in rats fed with the control or the GBF diet (Experiment 2). The data are expressed as mean changes $\pm \mathrm{SE}$. The butyrate and acetate content was significantly higher in the GBF group than that of the control. ${ }^{*} \mathrm{P}<0.05$ for the difference between the control and GBF.

that GBF had the potency to cause anti-colitis effects in HLAB27 transgenic rats which showed mild colitis along with hyperplasia in their colonic mucosa (34). Interestingly, GBF increased the mucosal barrier function in healthy animal specimens without hyperplasia. This effect of GBF may definitely be different between healthy subjects and those with certain pathogenic conditions.

The present study shows that prebiotic treatment (GBF) increased cecal $\beta$-glucosidase activity, while its $ß$-glucuronidase level remained the same as in the control group.
Although $\beta$-glucosidase activity is controversial, it was reported that inulin or the mixture of inulin and bifidobacterium longum significantly increases the activity in AOM-treated rats (35). In addition, some oligosaccharides have been shown to increase $\beta$-glucosidase activity in gut flora due to an increase in the amount of lactobacillus (36). This activity is reported to induce butyrate production, but its effect is unclear as this same activity has been reinforced in the generation of toxin and bacterial protective metabolites against chemically induced cancer.

The $\beta$-glucuronidase activity is reported to be closely related to carcinogenesis of AOM in the colon. Briefly, AOM is converted to methylazoxymethanol (MAM) by cytochrome P450 in the liver and is delivered to the colon, or MAM is conjugated with glucuronic acid in bile acid, and sequentially, it is hydrolyzed by bacterial B-glucuronidase and is absorbed in colon (37). In the two cases, MAM is further metabolized to a reactive compound to create the methylation of DNA. It was postulated that evidence of the same activity of B-glucuronidase between the two groups indicated that the active compound of carcinogenesis of AOM in the colon did not change its concentration. Thus, the preventive effect of GBF upon the incidence of preneoplastic action was not caused by the difference of MAM concentration in colon.

Slc5a8 is reported to be a solute carrier transporter which regulates the uptake of monocarboxylates in epithelium (15). Although $\sim 60 \%$ of short chain fatty acids (monocarboxylates) was absorbed by simple diffusion across the cell membranes, the residual $40 \%$ was absorbed by the ionized SCFA involving the co-transportation of inorganic protons, such as $\mathrm{Na}^{+}, \mathrm{K}^{+}$and $\mathrm{H}^{+}(23,24)$. In addition, it was reported that the high frequency of slc5a8 methylation observed in colon cancer and the

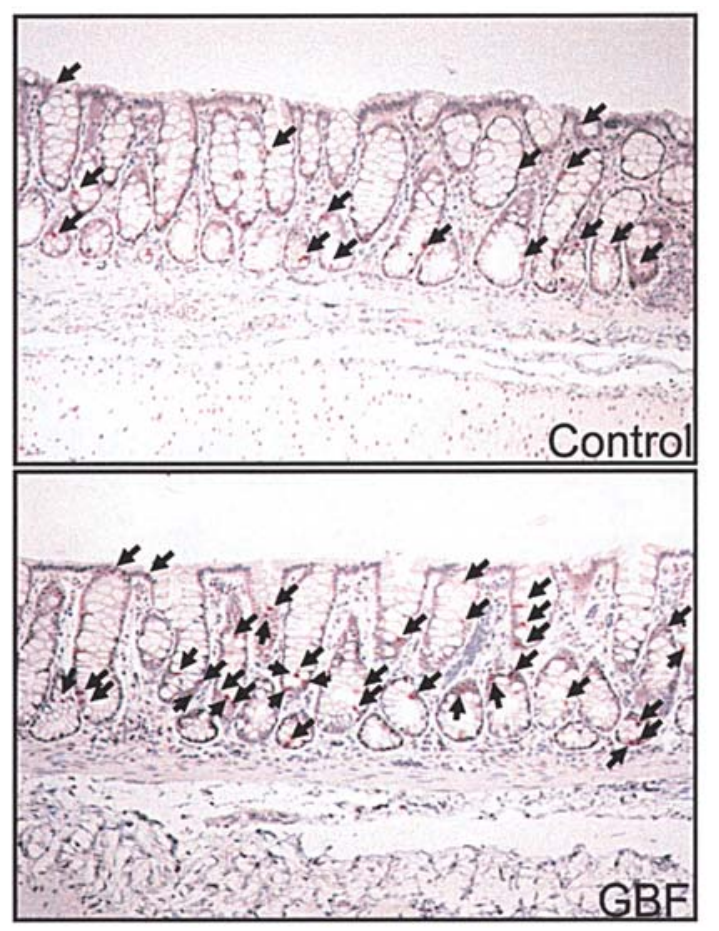

\section{HSP25 positive cells / view}

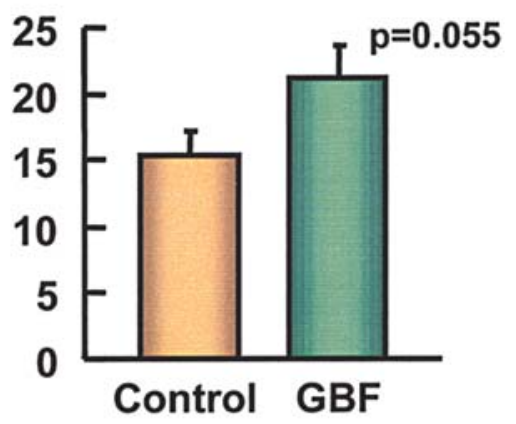

Figure 11. The arrows in the left panel show heat shock protein 25 (HSP25)-positive cells (at x200 magnification). The right panel shows the number of stained cells per view counted from 6 different areas ( 2 views in the distal, middle and proximal parts of the colon, respectively). The number of HSP25 in the GBF group was higher than that of the control group, although it did not reach a significant difference $(\mathrm{p}=0.055)$. The data are expressed as mean changes \pm SE. 
reconstruction of unmethylated slc5a8 in colon cancer cells suppressed the colony forming ability (14). In the present study, the increase of slc5a8 was observed in the colonic epithelium in the GBF group, although it did not reach a significant difference. Although more detailed studies are required, prebiotic treatment modulated the intestinal environment, and as a result, increased the butyrate content in the lower intestinal tract. Simultaneously, this change may produce an increased utilization of monocarboxylate in the epithelium and suppress the incidence of carcinogenesis through activation of the tumor suppressor gene slc5a8.

ß-catenin, acts as a scaffolding protein at cell-cell adherent junctions and as a transcriptional activator mediating Wnt signal transduction (38). Studies of B-catenin have also reported that frequent mutations were observed in AOMinduced colon cancer in rodent models and showed increased expression in colonic cancer models and in humans (39). In this study, the GBF treatment significantly attenuated the expression of $\beta$-catenin in colonic mucosa, in comparison with the control group. Kim et al suggested that a reduction of $\beta$-catenin by food alternation caused a decrease in the transcriptional activity stimulating $\beta$-catenin/T cell factor 4 responsive oncogenes such as c-myc (8). Although the complex effects of GBF on B-catenin are still unknown, when considering the evidence along with the data showing a decrease of ACF in GBF treatments, it can be postulated that GBF plays a pivotal role in the prevention of colon carcinogenesis development in the early stages.

Small heat shock protein, especially HSP25, has been suggested to confer a degree of mucosal protection against a number of injurious events (hyperthermia, ionizing radiation, and oxidative stress). HSP25 is also inducible by a variety of stimuli, such as cytokines, change in microbiota, and SCFA (27). It was reported that dietary-resistant starch prevented tumor induction concomitant in the case of increased colonic epithelium HSP25 formations in rats (40). A controversial report stated that HSP25 enhanced the tumorigenicity in immunogenic cell clones due to an increase of resistance to the apoptotic effect (41). In this study, GBF increased the formation of HSP25 and butyrate production in AOM-treated rats. The increase of HSP25 was considered to be induced by a change in the intestinal environment (butyrate). Furthermore, HSP25 may act to protect against the injurious damage caused by AOM. AOM treatment caused transient body weight loss in the two groups, making it evident that this treatment caused relatively severe stress to rats. Further in vivo studies regarding the role HSP25 plays concerning the pathogenesis of colon cancer are needed.

It is known that the risk of colorectal cancer is higher in patients with UC, compared to the general population $(42,43)$. However, not all UC patients have the same risk; a greater risk is observed in UC patients with more extensive colorectal inflammation which continues for longer periods. It is well known that UC is required for long-term medical and nutritional treatment to sustain the remission periods of patients. In conclusion, GBF showed a preventive effect on tumorigenicity in the AOM rat model. This result will contribute to the reduction of the risk of contracting colonic cancer in patients with IBD as well as in healthy humans (44). The nutraceutical preventive treatment of using GBF for colon cancer may prove useful without causing the adverse effects seen in treatments with anti-cancer or anti-inflammatory drugs.

\section{Acknowledgements}

We are grateful to Miss Tomoko Nii (Kirin Holdings Co. Ltd.) for her excellent technical assistance.

\section{References}

1. Guarner F, Casellas F, Borruel N, Antolín M, Videla S, Vilaseca J and Malagelada JR: Role of microecology in chronic inflammatory bowel diseases. Eur J Clin Nutr 56 (Suppl 4): S34-S38, 2002.

2. Shiba T, Aiba Y, Ishikawa H, Ushiyama A, Takagi A, Mine T and Koga Y: The suppressive effect of bifidobacteria on Bacteroides vulgatus, a putative pathogenic microbe in inflammatory bowel disease. Microbiol Immunol 47: 371-378, 2003.

3. Nakanishi S, Kataoka K, Kuwahara T and Ohnishi Y: Effects of high amylose maize starch and Clostridium butyricum on metabolism in colonic microbiota and formation of azoxymethaneinduced aberrant crypt foci in the rat colon. Microbiol Immunol 47: 951-958, 2003.

4. Le Leu RK, Brown IL, Hu Y, Bird AR, Jackson M, Esterman A and Young GP: A synbiotic combination of resistant starch and Bifidobacterium lactis facilitates apoptotic deletion of carcinogen-damaged cells in rat colon. J Nutr 135: 996-1001, 2005.

5. Park E, Jeon GI, Park JS and Paik HD: A probiotic strain of Bacillus polyfermenticus reduces DMH induced precancerous lesions in F344 male rat. Biol Pharm Bull 30: 569-574, 2007.

6. Hanai H, Kanauchi O, Mitsuyama K, Andoh A, Takeuchi K, Takayuki I, Araki Y, Fujiyama Y, Toyonaga A, Sata M, Kojima A, Fukuda $\mathrm{M}$ and Bamba T: Germinated barley foodstuff prolongs remission in patients with ulcerative colitis. Int J Mol Med 13: 643-647, 2004.

7. Mitsuyama K, Saiki T, Kanauchi O, Iwanaga T, Tomiyasu N, Nishiyama T, Tateishi H, Shirachi A, Ide M, Suzuki A, Noguchi K, Ikeda $\mathrm{H}$, Toyonaga A and Sata M: Treatment of ulcerative colitis with germinated barley foodstuff feeding: a pilot study. Aliment Pharmacol Ther 12: 1225-1230, 1998.

8. Kim YS and Milner JA: Dietary modulation of colon cancer risk. J Nutr 137: S2576-S2579, 2007.

9. Comalada M, Bailón E, de Haro O, Lara-Villoslada F, Xaus J, Zarzuelo A and Gálvez J: The effects of short-chain fatty acids on colon epithelial proliferation and survival depend on the cellular phenotype. J Cancer Res Clin Oncol 132: 487-497, 2006.

10. Hinnebusch BF, Meng S, Wu JT, Archer SY and Hodin RA: The effects of short-chain fatty acids on human colon cancer cell phenotype are associated with histone hyperacetylation. J Nutr 132: 1012-1017, 2002.

11. Caderni G, Luceri C, De Filippo C, Salvadori M, Giannini A, Tessitore L and Dolara P: Slow-release pellets of sodium butyrate do not modify azoxymethane (AOM)-induced intestinal carcinogenesis in F344 rats. Carcinogenesis 22: 525-527, 2001.

12. Caderni G, Luceri C, Lancioni L, Tessitore L and Dolara P: Slow-release pellets of sodium butyrate increase apoptosis in the colon of rats treated with azoxymethane, without affecting aberrant crypt foci and colonic proliferation. Nutr Cancer 30: $175-181,1998$.

13. D'Argenio G, Cosenza V, Delle Cave M, Iovino P, Delle Valle N, Lombardi G and Mazzacca G: Butyrate enemas in experimental colitis and protection against large bowel cancer in a rat model. Gastroenterology 110: 1727-1734, 1996.

14. Li H, Myeroff L, Smiraglia D, Romero MF, Pretlow TP, Kasturi L, Lutterbaugh J, Rerko RM, Casey G, Issa JP, Willis J, Willson JK, Plass C and Markowitz SD: SLC5A8, a sodium transporter, is a tumor suppressor gene silenced by methylation in human colon aberrant crypt foci and cancers. Proc Natl Acad Sci USA 100: 8412-8417, 2003.

15. Miyauchi S, Gopal E, Fei YJ and Ganapathy V: Functional identification of SLC5A8, a tumor suppressor down-regulated in colon cancer, as a $\mathrm{Na}(+)$-coupled transporter for short-chain fatty acids. J Biol Chem 279: 13293-13296, 2004.

16. Gupta N, Martin PM, Prasad PD and Ganapathy V: SLC5A8 (SMCT1)-mediated transport of butyrate forms the basis for the tumor suppressive function of the transporter. Life Sci 78: 2419-2425, 2006. 
17. Nozawa H, Yoshida A, Tajima O, Katayama M, Sonobe H, Wakabayashi $\mathrm{K}$ and Kondo K: Intake of beer inhibits azoxymethane-induced colonic carcinogenesis in male Fischer 344 rats. Int J Cancer 108: 404-411, 2004.

18. Plate AY and Gallaher DD: Effects of indole-3-carbinol and phenethyl isothiocyanate on colon carcinogenesis induced by azoxymethane in rats. Carcinogenesis 27: 287-292, 2006.

19. Wali RK, Khare S, Tretiakova M, Cohen G, Nguyen L, Hart J, Wang J, Wen M, Ramaswamy A, Joseph L, Sitrin M, Brasitus T and Bissonnette M: Ursodeoxycholic acid and F(6)-D(3) inhibit aberrant crypt proliferation in the rat azoxymethane model of colon cancer: roles of cyclin D1 and E-cadherin. Cancer Epidemiol Biomarkers Prev 11: 1653-1662, 2002.

20. Kanauchi $\mathrm{O}$ and Agata K: Protein, and dietary fiber-rich new foodstuff from brewer's spent grain increased excretion of feces and jejunum mucosal protein content in rats. Biosci Biotechnol Biochem 61: 29-33, 1997.

21. Kanauchi O, Nakamura T, Agata K, Mitsuyama K and Iwanaga T: Effects of germinated barley foodstuff on dextran sulfate sodium-induced colitis in rats. J Gastroenterol 33: 179-188, 1998.

22. Araki Y, Andoh A, Koyama S, Fujiyama Y, Kanauchi O and Bamba T: Effects of germinated barley foodstuff on microflora and short chain fatty acid production in dextran sulfate sodiuminduced colitis in rats. Biosci Biotechnol Biochem 64: 1794-1800, 2000.

23. Takebe K, Nio J, Morimatsu M, Karaki S, Kuwahara A, Kato I and Iwanaga T: Histochemical demonstration of a $\mathrm{Na}(+)$-coupled transporter for short-chain fatty acids (slc5a8) in the intestine and kidney of the mouse. Biomed Res 26: 213-221, 2005.

24. Iwanaga T, Takebe K, Kato I, Karaki S and Kuwahara A: Cellular expression of monocarboxylate transporters (MCT) in the digestive tract of the mouse, rat, and humans, with special reference to slc5a8. Biomed Res 27: 243-254, 2006.

25. Fujise T, Iwakiri R, Kakimoto T, Shiraishi R, Sakata Y, Wu B, Tsunada S, Ootani A and Fujimoto K: Long-term feeding of various fat diets modulates azoxymethane-induced colon carcinogenesis through Wnt/beta-catenin signaling in rats. Am J Physiol Gastrointest Liver Physiol 292: G1150-G1156, 2007.

26. Djouzi Z, Andrieux C, Degivry MC, Bouley C and Szylit O: The association of yogurt starters with Lactobacillus casei DN 114.001 in fermented milk alters the composition and metabolism of intestinal microflora in germ-free rats and in human floraassociated rats. J Nutr 127: 2260-2266, 1997.

27. Ren H, Musch MW, Kojima K, Boone D, Ma A and Chang EB: Short-chain fatty acids induce intestinal epithelial heat shock protein 25 expression in rats and IEC 18 cells. Gastroenterology 121: 631-639, 2001.

28. Kanauchi O, Iwanaga T, Mitsuyama K, Saiki T, Tsuruta O, Noguchi $\mathrm{K}$ and Toyonaga A: Butyrate from bacterial fermentation of germinated barley foodstuff preserves intestinal barrier function in experimental colitis in the rat model. J Gastroenterol Hepatol 14: 880-888, 1999.

29. Kanauchi O, Iwanaga T, Andoh A, Araki Y, Nakamura T, Mitsuyama K, Suzuki A, Hibi T and Bamba T: Dietary fiber fraction of germinated barley foodstuff attenuated mucosal damage and diarrhea, and accelerated the repair of the colonic mucosa in an experimental colitis. J Gastroenterol Hepatol 16: 160-168, 2001.
30. Kanauchi O, Serizawa I, Araki Y, Suzuki A, Andoh A, Fujiyama Y, Mitsuyama K, Takaki K, Toyonaga A, Sata M and Bamba T: Germinated barley foodstuff, a prebiotic product, ameliorates inflammation of colitis through modulation of the enteric environment. J Gastroenterol 38: 134-141, 2003.

31. Guarner F: Enteric flora in health and disease. Digestion 73 (Suppl 1): 5-12, 2006.

32. Perrin P, Pierre F, Patry Y, Champ M, Berreur M, Pradal G, Bornet F, Meflah K and Menanteau J: Only fibres promoting a stable butyrate producing colonic ecosystem decrease the rate of aberrant crypt foci in rats. Gut 48: 53-61,2001.

33. Jänne PA and Mayer RJ: Chemoprevention of colorectal cancer. N Engl J Med 342: 1960-1968, 2000.

34. Kanauchi O, Andoh A, Iwanaga T, Fujiyama Y, Mitsuyama K, Toyonaga A and Bamba T: Germinated barley foodstuffs attenuate colonic mucosal damage and mucosal nuclear factor kappa B activity in a spontaneous colitis model. J Gastroenterol Hepatol 14: 1173-1179, 1999.

35. Rowland IR, Rumney CJ, Coutts JT and Lievense LC: Effect of Bifidobacterium longum and inulin on gut bacterial metabolism and carcinogen-induced aberrant crypt foci in rats. Carcinogenesis 19: 281-285, 1998.

36. Rowland IR and Tanaka R: The effects of transgalactosylated oligosaccharides on gut flora metabolism in rats associated with a human faecal microflora. J Appl Bacteriol 74: 667-674, 1993.

37. Suaeyun R, Kinouchi T, Arimochi H, Vinitketkumnuen U and Ohnishi Y: Inhibitory effects of lemon grass (Cymbopogon citratus Stapf) on formation of azoxymethane-induced DNA adducts and aberrant crypt foci in the rat colon. Carcinogenesis 18: 949-955, 1997.

38. Sena P, Saviano M, Monni S, Losi L, Roncucci L, Marzona L and De Pol A: Subcellular localization of beta-catenin and APC proteins in colorectal preneoplastic and neoplastic lesions. Cancer Lett 241: 203-212, 2006.

39. Kohno H, Suzuki R, Sugie S and Tanaka T: Beta-catenin mutations in a mouse model of inflammation-related colon carcinogenesis induced by 1,2-dimethylhydrazine and dextran sodium sulfate. Cancer Sci 96: 69-76, 2005.

40. Bauer-Marinovic M, Florian S, Müller-Schmehl K, Glatt H and Jacobasch G: Dietary resistant starch type 3 prevents tumor induction by 1,2-dimethylhydrazine and alters proliferation, apoptosis and dedifferentiation in rat colon. Carcinogenesis 27: 1849-1859, 2006.

41. Lee YJ, Lee DH, Cho CK, Chung HY, Bae S, Jhon GJ, Soh JW, Jeoung DI, Lee SJ and Lee YS: HSP25 inhibits radiationinduced apoptosis through reduction of PKCdelta-mediated ROS production. Oncogene 24: 3715-3725, 2005.

42. Eaden JA, Abrams KR and Mayberry JF: The risk of colorectal cancer in ulcerative colitis: a meta-analysis. Gut 48: 526-535, 2001.

43. Rutter MD, Saunders BP, Wilkinson KH, Rumbles S, Schofield G, Kamm MA, Williams CB, Price AB, Talbot IC and Forbes A: Cancer surveillance in longstanding ulcerative colitis: endoscopic appearances help predict cancer risk. Gut 53: 1813-1816, 2004.

44. Geier MS, Butler RN and Howarth GS: Probiotics, prebiotics and synbiotics: a role in chemoprevention for colorectal cancer? Cancer Biol Ther 5: 1265-1269, 2006. 